\title{
INTERCÂMBIO INTERNACIONAL EEAN/UFRJ E INSTITUTO SUPERIOR DE CIÊNCIAS DA SAÚDE DA REPÚBLICA DE MOÇAMBIQUE: CRIANDO AS BASES DE UMA COOPERAÇÃO TÉCNICO-EDUCACIONAL ${ }^{a}$
}

\author{
International Exchange between EEAN/ UFRJ and ISCISA/ Mozambique: \\ creating the basis of a technical-educational cooperation \\ Intercambio Internacional entre EEAN/ UFRJ e ISCISA/ Mozambique: \\ creando las bases de una cooperación técnico-educacional
}

Marléa Chagas Moreira

Ivone Evangelista Cabral
Neide Aparecida Titonelli Alvim

Lidia Monjane
Maria Antonieta Rubio Tyrrell

Domingos E. Tuto

\begin{abstract}
Resumo
0 estudo visa descrever as atividades desenvolvidas durante visita de trabalho de uma delegação do Instituto Superior de Ciências da Saúde da República de Moçambique (ISCISA) na Escola de Enfermagem Anna Nery/UFRJ, bem como as primeiras repercussões que culminaram em proposta de convênio entre estas instituições. 0 propósito foi o estabelecimento de cooperação e intercâmbio internacional para formação e qualificação de recursos humanos em enfermagem. Como resultado dessa visita, foi assinada uma Carta de Intenção entre EEAN/UFRJ e ISCISA, cujas primeiras repercussões foi a realização de uma Reunião Científica no Brasil e uma Missão Brasileira de cooperação técnico-educacional em Moçambique.
\end{abstract}

Palavras-chave: Enfermagem. Intercâmbio Educacional Internacional. Pesquisa.

\section{Abstract}

The study aims to describe the activities developed during the visit of work from a delegation of the Superior Institute of Health Sciences from the Republic of Mozambique (ISCISA), in the Anna Nery School of Nursing/UFRJ, as well as the first repercussions that had culminated in an agreement proposal between these institutions. The intention was the establishment of cooperation and international exchange for formation and qualification of human resources in nursing. As result of this visit was signed a Letter of Intention between EEAN/UFRJ and ISCISA, whose first repercussions were the accomplishment of a Scientific Meeting in Brazil and a Brazilian Mission of technical-educational cooperation in Mozambique.

Keywords:

Nursing. International Educational Exchange. Research.

\section{Resumen}

El estudio visa describir las actividades desarrolladas durante la visita de trabajo de una delegación del Instituto Superior de las Ciencias de la Salud de la República de Mozambique (ISCISA), en la Escuela de Enfermería Ana Nery/UFRJ, así como las primeras repercusiones que habían culminado en una oferta de acuerdo entre estas instituciones. La intención era el establecimiento de la cooperación y del intercambio internacional para la formación y calificación de recursos humanos en enfermería. Como resultado de esta visita fue firmada una Carta de Intención entre EEAN/UFRJ e ISCISA, cuyas primeras repercusiones fueron la realización de una Reunión Científica en el Brasil y una Misión Brasileña de Cooperación Tecnico-Educacional en Mozambique.

\section{Palabras clave:}

Enfermería. Intercambio Educacional Internacional. Investigación. 


\section{O PONTO DE PARTIDA}

Dentro do contexto de reconfiguração do cenário geopolítico mundial no início do terceiro milênio, uma nova ordem tem sido direcionada pela reestruturação das políticas internacionais. Nesse sentido, um grande desafio foi lançado pela Organização das Nações Unidas, em 2002, aos diversos países, com vistas ao alcance de metas que objetivam 0 desenvolvimento social e econômico, principalmente nas áreas de saúde, ciência e tecnologia. Isto implica o desenvolvimento de estratégias que favoreçam as parcerias e intercâmbios cultural, tecnológico, empresarial e acadêmico ${ }^{1}$.

A parceria entre a EEAN e os países africanos no âmbito da formação profissional já vem de longa data. Na pós-graduação lato sensu, registra-se o Curso de Aperfeiçoamento em Ensino de Enfermagem para seis enfermeiros docentes de Maputo Moçambique, caracterizando-se como um Programa de Cooperação Técnica e Intercâmbio Internacional BrasilMoçambique e, mais especificamente, entre a Universidade Federal do Rio de Janeiro/Escola de Enfermagem Anna Nery e a Direção de Formação e Treinamento do Ministério da Saúde de Moçambique. 0 curso, realizado no período de maio a julho de 1989, com carga horária global de 360 horas, foi coordenado pelas professoras Maria Antonieta Rubio Tyrrel, Dely Gonçalves de Oliveira e Suely de Souza Baptista ?

A partir dessa primeira experiência, a EEAN, no contexto da missão da UFRJ de favorecer o fortalecimento da Unidade Nacional e a solidariedade internacional ${ }^{3}$, tem participado de projetos educacionais para formação profissional. No curso de graduação, recebemos sete estudantes de Angola no período de 1994 a 2004, dos quais dois estão com o curso em andamento. As vagas são oferecidas através de Convênio Cultural/Cortesia da Universidade Federal do Rio de Janeiro ${ }^{4}$, integrando o Programa Estudante-Convênio (PEC), celebrado entre o Departamento de Cooperação Científica, Técnica e Tecnológica do Ministério das Relaç̃̃es Exteriores e a Coordenação de Aperfeiçoamento de Pessoal de Nível Superior (CAPES).

\section{ESTREITANDO OS VÍNCULOS}

Em setembro de 2004, a Direção Pedagógica do Instituto Superior de Ciências da Saúde (ISCISA) solicitou à Direção da EEAN/UFRJ autorização para realização de visita de trabalho de uma delegação daquela instituição constituída pelo Profo Dr. Domingos E. Tuto, subdiretor do ISCISA; Profa Lidia Monjane, diretora pedagógica, e Profo Marcos JoséCanda, diretor do Curso de Licenciatura em Enfermagem, visando conhecer o processo administrativo-pedagógico da EEAN, assim como estabelecer bases para uma cooperação técnicoeducacional. 0 ISCISA é uma instituição estatal de ensino superior localizada em Maputo. Iniciou as atividades acadêmicas em março de 2004, nas áreas de Enfermagem Geral, Enfermagem de Saúde Materna e Cirurgia. Em 2005, ampliou esta formação com previsão de abertura dos Cursos de Tecnologia Biomédica e Laboratório e Enfermagem Pediátrica, entre outros.

Atendendo à solicitação, a visita foi realizada no período de 01 a 14 de outubro de 2004. No marco desta visita, a Direção e a Coordenação de Pós-Graduação e Pesquisa da EEAN, através da Coordenação Adjunta de Aperfeiçoamento Docente e Discente, coordenada pela Prof $^{\mathrm{a}} \mathrm{D} \mathrm{r}^{\mathrm{a}}$ Marléa Chagas Moreira, organizaram o evento "Programa de Aperfeiçoamento Docente e Gestão Acadêmica", realizado no período de 04 a 15 de outubro de 2004.

Este evento teve como finalidades: apresentar a estrutura organizacional da EEAN e suas estratégias de ação nacional e internacional; apresentar as estratégias de gestão acadêmica com articulação ensino, pesquisa e extensão; partihar experiências do processo ensino-aprendizagem; apresentar a Pós-Graduação da EEAN e as diferentes formas de articulação com a Graduação; conhecer as expectativas do grupo moçambicano em relação à EEAN; e discutir as diferentes possibilidades de intercâmbio.

As atividades contempladas neste evento foram: reunião com a diretora da EEAN com a participação das coordenadoras dos cursos de Graduação, Pós-Graduação, Extensão, Licenciatura e Programas Curriculares Interdepartamentais do Curso de Graduação; visitas às dependências da PósGraduação, do Comitê de Ética em Pesquisa, da Coordenação de Graduação, do Centro de Documentação, além de visita a campos de estágio do curso de graduação: Hospital Universitário Clementino Fraga Filho, Instituto de Pediatria Martagão Gesteira, Hospital Escola São Francisco de Assis. Foram também realizadas visitas à Faculdade de Enfermagem da Universidade Estadual do Rio de Janeiro e à Fundação Oswaldo Cruz - Manguinhos.

A partir das discussões realizadas no âmbito do mencionado evento, foi elaborada uma Carta de Intenções entre a EEAN e ISCISA, que destaca, entre outros, os seguintes pontos: assessoria e consultoria nas áreas acadêmicas e de gestão; implementação de Programas Especiais Pedagógicos Bilaterais no âmbito da graduação e pós-graduação; intercâmbio de pesquisadores, de investigações multicêntricas e de publicações, na perspectiva internacional, através de revistas institucionais; oferta de Programa de Capacitação Docente; intercâmbio de estudantes de graduação, mestrado e doutorado; oferta de estágios de desenvolvimento em nível de graduação e pós-graduação; e promoção de eventos técnico-científicos de interesse comum às instituições envolvidas.

\section{AS PRIMEIRAS REPERCUSSÕES}

0 Programa de Aperfeiçoamento Docente e Gestão Acadêmica teve seus objetivos plenamente alcançados, de acordo com a avaliação realizada pela delegação moçambicana. As primeiras repercussões do intercâmbio EEAN/ISCISA se concretizaram na realização de Reunião Científica no Brasil e de Missão de trabalho de docentes da EEAN em Moçambique, visando atender às necessidades mais imediatas da equipe moçambicana, no âmbito da formação profissional.

\section{Reunião Científica Formação Profissional em Enfermagem: Brasil - Moçambique}

0 evento realizado no dia 14 de outubro de 2004, no Pavilhão de Aulas da EEAN, foi promovido pelas Coordenações Adjunta de Aperfeiçoamento Docente e Discente, e de Graduação em Enfermagem, e pelo Núcleo de Pesquisa em Educação, Gerência e Exercício Profissional da Enfermagem 
(NUPEGEPEn) da EEAN. Os objetivos foram: proporcionar intercâmbio entre docentes e estudantes sobre questões relacionadas à formação profissional de enfermagem no Brasil e em Moçambique, e discutir possibilidades de parcerias para o avanço da formação profissional em enfermagem entre esses países.

0 evento contou com 48 participantes: 21 docentes, 14 estudantes de graduação e 13 de pós-graduação stricto sensu da EEAN, alguns dos quais representando suas instituições de origem, assistenciais ou educacionais do Rio de Janeiro.

A programação incluiu os seguintes temas: "Exercício da Enfermagem em Moçambique: desafios e perspectivas para a formação profissional", proferido pelo Dr. Domingos Tuto (ISCISA) e "Diretrizes curriculares para a formação de Enfermagem: o nível médio e a graduação no Brasil" proferido pela Dra Lígia de Oliveira Viana e Enfermeira Gesilda Soraia Cavalcanti Valente, pesquisadora e mestranda, do NUPEGEPEn/EEAN, respectivamente. As apresentações foram seguidas de amplo debate.

Durante o evento foi configurada cerimônia para assinatura da Carta de Intenções entre a EEAN e o ISCISA, selando as bases de uma cooperação técnico-educacional entre as instituições nas áreas de interesse moçambicano para assessoria e consultoria para formação de profissionais de enfermagem, gestão de cursos e participação em programas pedagógico-acadêmicos bilaterais, investigação e divulgação da informação, intercâmbio de estudantes e programas de extensão.

\section{Missão de Trabalho em Moçambique}

Como primeira missão do intercâmbio em Moçambique, em dezembro de 2004, a EEAN recebeu solicitação do Dr. Aurélio Amândio Zilhão, diretor do ISCISA, para que a Escola enviasse uma delegação a Moçambique para prestar assessoria e consultoria acadêmicas e de gestão na área de saúde materno-infantil, além de proferir magna conferência no Dia Internacional da Enfermagem. Esta missão foi realizada no período de 15 a 20 de maio de 2005, composta pelas professoras doutoras Maria Antonieta Rubio Tyrrell - diretora da EEAN, Neide Aparecida Titonelli Alvim - coordenadora geral de Pós-Graduação e Pesquisa, e lvone Evangelista Cabral - diretora do Núcleo de Pesquisa em Enfermagem em Saúde da Criança.

Por ocasião desta missão, com o objetivo de fortalecer o processo de implementação e desenvolvimento da carreira de Enfermagem em Moçambique e formação profissional do primeiro curso de Enfermagem e Enfermagem Pediátrica no ISCISA, este Instituto e a EEAN, de mútuo acordo, estabeleceram uma proposta de convênio, conforme as linhas de ação a seguir: A) assessoria e consultoria nas áreas acadêmicas e de gestão voltadas aos cursos de graduação em Enfermagem e Enfermagem Pediátrica de Formação Geral do ISCISA, com participação de docentes da EEAN na equipe de avaliação periódica dos currículos do ISCISA relativos à formação nessas áreas. B) implementação de programas especiais pedagógicos bilaterais no âmbito da graduação e pósgraduação através do desenvolvimento de eventos científicos; intercâmbios de estudantes e docentes através da oferta de estágios, promovendo condições para o encaminhamento de estudantes de ambos os países e ampliando as relações; participação na formação pedagógica; e especialização para o corpo docente do curso de Enfermagem Pediátrica oferecido pelo ISCISA. C) intercâmbio de pesquisadores e estabelecimento de investigações multicêntricas por meio de estudos científicos nas áreas de saúde da mulher, saúde da criança e saúde pública, através de protocolos multicêntricos Moçambique-Brasil, fundamentada na realidade epidemiológica dos dois países. D) intercâmbio de publicações na perspectiva internacional, através de revistas institucionais indexadas, com a promoção de permuta de publicações, visando à divulgação dos resultados das pesquisas multicêntricas de ambos os países e a visibilidade da realidade da Enfermagem em ambos os países. E) assessorias e consultorias temporárias em gestão acadêmica.

\section{CONSIDERAÇÕES FINAIS}

Acreditamos que a concretização deste intercâmbio foi um passo significativo para o ISCISA e reafirmou o compromisso da EEAN com a formação e capacitação dos profissionais de Enfermagem, inclusive no contexto internacional. Isso nos coloca diante de mais um desafio, considerando que, no contexto do processo de transformação vivenciado pela universidade brasileira, principalmente no que se refere à sua função histórica e social, o compromisso da EEAN é com a formação de pessoas com idéias e ações autônomas, de juízo crítico, e comprometidas com o pleno exercício da democracia, da solidariedade e da equidade social ${ }^{5}$.

No que tange às repercussões na pós-graduação, esta iniciativa vem ao encontro do preconizado pela Políica Nacional de Pós-Graduação quando estimula a busca de cooperação internacional, reforçando o seu caráter bilateral, ou seja, a possibilidade de prestar e receber cooperação de diferentes naturezas, a exemplo da produção científica e da aquisição de métodos e tecnologias de estudo e de trabalho.

0 propósito de apresentar os resultados alcançados com 0 intercâmbio é o de estimular outras Escolas de Enfermagem/ Programas de Pós-Graduação a estabelecer diferentes modalidades de intercâmbio e parcerias, além de demonstrar às agências de fomento as potencialidades das enfermeiras brasileiras no desenvolvimento de projetos educacionais, de capacitação profissional e de pesquisa, que contribuam para a formação de recursos humanos, produção e difusão do conhecimento que possam colaborar com melhoria da qualidade de vida nos diversos países. 


\section{Referências}

1. Morel CM. A pesquisa em saúde e os objetivos do milênio: desafios e oportunidades globais, soluções e políticas nacionais. Cienc Saúde Col 2004 abr/jun; 9 (2): 261-70.

2. Universidade Federal do Rio de Janeiro. Escola de Enfermagem Anna Nery. Coordenação Geral de Pós-Graduação e Pesquisa. Relatório do Curso de Aperfeiçoamento de Ensino de Enfermagem-1989. Rio de Janeiro (RJ);1989.

3. Universidade Federal do Rio de Janeiro. Estatuto da Universidade Federal do Rio de Janeiro. [acesso 10 out 2006]. Disponível em: // www.ufrj.br//pr/conteúdo_pr?sigla=ESTATUTO.

4. Resolução CEG/UFRJ nº 4 de 06 de julho de 1988. Dispõe sobre normas referentes a alunos cuja matrícula decorre de convênio cultural ou de cortesia. [on-line] [acesso 10 out 2006]. Disponíve em: http: / /www.pr1.ufrj.br/pr1/ceg/resoluções/default.php.

5. Tyrrell MAR. Desafios da enfermagem e perspectivas de ação da EEAN. Discurso. Esc Anna Nery Rev Enferm 2002 abr; 6(1): 23-7.

\section{Notas}

Trabalho associado ao intercâmbio EEAN/UFRJ e Instituto Superior de Ciências da Saúde da República de Moçambique (ISCISA/ Moçambique) no marco da assinatura da Carta de Intenções.
bMarcos José Canda - Diretor do Curso de Licenciatura em Enfermagem do ISCISA/Moçambique. Por ter integrado a delegação da ISCISA que veio pleitear o Convênio entre a EEAN/UFRJ e ISCISA em outubro de 2004. E por ter participado das discussões acerca dos termos do convênio em maio de 2005, Moçambique.

\section{Sobre os Autores}

\section{Marléa Chagas Moreira}

Professora Adjunta do Departamento de Metodologia da Enfermagem. Coordenadora de Aperfeiçoamento Docente e Discente EEAN/UFRJ.

\section{Neide Aparecida Titonelli Alvim}

Professora Adjunta do Departamento de Enfermagem Fundamental. Coordenadora dos Cursos de Pós-Graduação e Pesquisa da EEAN/UFRJ.

\section{Maria Antonieta Rubio Tyrrell}

Professora Titular do Departamento de Enfermagem Materno-Infantil. Diretora da EEAN/UFRJ.

\section{Ivone Evangelista Cabral}

Professora Associada do Departamento de Enfermagem Materno-Infantil.

\section{Lidia Monjane}

Mestre em Ciências de Educação. Diretora Pedagógica do ISCISA/Moçambique

Domingos E. Tuto

Vice Diretor ISCISA. 